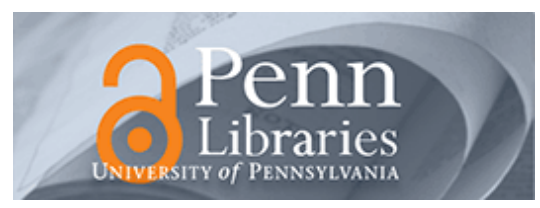

University of Pennsylvania

ScholarlyCommons

$11-14-2007$

\title{
Continuum Theory for Piezoelectric Response of Chiral Nanotubes Under Uniaxial and Torsional Stresses
}

Paul Joseph Michalski

University of Pennsylvania

Eugene J. Mele

University of Pennsylvania, mele@physics.upenn.edu

Follow this and additional works at: https://repository.upenn.edu/physics_papers

Part of the Physics Commons

\section{Recommended Citation}

Michalski, P., \& Mele, E. J. (2007). Continuum Theory for Piezoelectric Response of Chiral Nanotubes Under Uniaxial and Torsional Stresses. Retrieved from https://repository.upenn.edu/physics_papers/121

Suggested Citation:

P.J. Michalski and E.J. Mele. (2007). "Continuum theory for piezoelectric response of chiral nanotubes under uniaxial and torsional stresses." Physical Review B. 76, 205419.

(C) 2007 The American Physical Society

http://www.dx.doi.org/10.1103/PhysRevB.76.205419

This paper is posted at ScholarlyCommons. https://repository.upenn.edu/physics_papers/121

For more information, please contact repository@pobox.upenn.edu. 


\title{
Continuum Theory for Piezoelectric Response of Chiral Nanotubes Under Uniaxial and Torsional Stresses
}

\author{
Abstract \\ We develop and solve a continuum theory for the piezoelectric response of nanotubes under applied \\ uniaxial and torsional stresses. We find that the piezoelectric response is controlled by the chiral angle, \\ the aspect ration, and two dimensionless parameters specifying the ratio of the strengths of the \\ electrostatic and elastic energies. The model is solved in two limiting cases and the solutions are \\ discussed. These systems are found to have several unxpected physical effects not seen in conventional \\ bulk systems, including a strong stretch-twist coupling and the development of a signficant bound charge \\ density in addition to a surface charge density. \\ Disciplines \\ Physical Sciences and Mathematics | Physics

\section{Comments} \\ Suggested Citation: \\ P.J. Michalski and E.J. Mele. (2007). "Continuum theory for piezoelectric response of chiral nanotubes \\ under uniaxial and torsional stresses." Physical Review B. 76, 205419. \\ (C) 2007 The American Physical Society \\ http://www.dx.doi.org/10.1103/PhysRevB.76.205419
}




\title{
Continuum theory for piezoelectric response of chiral nanotubes under uniaxial and torsional stresses
}

\author{
P. J. Michalski and E. J. Mele \\ Department of Physics and Astronomy, University of Pennsylvania, Philadelphia, Pennsylvania 19104, USA
}

(Received 10 August 2007; published 14 November 2007)

\begin{abstract}
We develop and solve a continuum theory for the piezoelectric response of nanotubes under applied uniaxial and torsional stresses. We find that the piezoelectric response is controlled by the chiral angle, the aspect ratio, and two dimensionless parameters specifying the ratio of the strengths of the electrostatic and elastic energies. The model is solved in two limiting cases and the solutions are discussed. These systems are found to have several unexpected physical effects not seen in conventional bulk systems, including a strong stretch-twist coupling and the development of a significant bound charge density in addition to a surface charge density. The model is applied to estimate the piezoelectric response of a boron nitride nanotube under uniform tensile stress.
\end{abstract}

DOI: 10.1103/PhysRevB.76.205419

PACS number(s): 73.63.Fg, 73.63.Bd, 77.65.Ly, 61.46.Fg

\section{INTRODUCTION}

Nanotubes and nanowires are a family of one dimensional structures whose properties can be tailored by their chemical composition, size, and shape. The lateral dimensions of these structures can be reduced to the nanometer scale, a feature that is exploited in almost all applications envisioned for these objects. Carbon nanotubes are the prototypical member of this family; they are the simplest chemically and are known to exhibit a diversity of electronic behaviors that are determined by the boundary conditions imposed on their electronic wave functions when they are mapped onto a cylindrical surface. While this sensitivity makes for rich theoretical study, it also makes carbon nanotubes (CNTs) difficult to use in applications since tubes must be identified and sorted according to their lattice structures. Boron nitride nanotubes (BNNTs) are the III-V analog of CNTs and are an attractive alternative to CNTs because they have similar mechanical properties, are chemically inert over a wide range of conditions, and, importantly, are all semiconducting with a band gap that is nearly independent of the chirality.

A BN nanotube is a heteropolar structure with a lower lattice symmetry than the carbon nanotube. Importantly, a twofold rotation about a perpendicular axis through the tube interchanges its $A$ and $B$ sublattices; this is a symmetry operation of the carbon nanotube but not of the boron nitride tube. In early theoretical work, it was appreciated that this allows for a nonzero electric polarization in the ground state of the heteropolar tube ${ }^{1}$ and a linear coupling of the polarization to a uniform strain, ${ }^{2}$ i.e., $\mathrm{BN}$ nanotubes are piezoelectric. ${ }^{3}$ Interestingly, the piezoelectricity is a property inherited from the flat BN sheet, while the electric polarization of an unstrained tube is a new property that only occurs when the sheet is wrapped to form a nanotube.

The description of piezoelectricity in a one dimensional nanotube differs fundamentally from the analogous formulation for a three dimensional crystal. In an ordinary three dimensional piezoelectric material described within the standard Landau-Devonshire theory, its macroscopic polarization (a vector) is linearly coupled to a uniform strain field (a tensor) through a third-rank piezoelectric tensor. Physically, this reflects the fact that bound charges arise from a diver- gence of the polarization, and these bound charges are mutually electrostatically coupled by the Coulomb interaction which diverges at small momentum proportional to $1 / q^{2}$. Thus, the free energy contains a term that is bilinear in the polarization that remains constant in the long wavelength $q \rightarrow 0$ limit. As a consequence, a uniformly strained three dimensional piezoelectric induces a state of uniform bulk polarization and the bound charges reside precisely at the sample boundaries.

This state of affairs is upset for a one dimensional piezoelectric because the Coulomb potential that couples the bound charges has only a softer $-\log q$ long wavelength divergence in one dimension. Thus, the analogous electrostatic coupling bilinear in the polarization vanishes in the long wavelength limit. The consequences of this are quite interesting and were partially explored in our previous work. ${ }^{4}$ In essence, the strain and polarization must be treated as inhomogeneous fields, whose equilibrium forms can be obtained by minimization of an appropriate free energy that contains a nonlocal coupling between the one dimensional strain gradients. As a consequence, a uniform applied mechanical stress generally induces a state of nonuniform strain in a nanotube or nanowire, and the bound charge redistributes from the tube end into its interior in a self-consistent fashion. The distribution of the equilibrium strain and polarization fields depends on a subtle interplay of their elastic and electrostatic interactions. Experimentally, available nanotubes are expected to fall in a parameter regime where the elastic interactions dominate the problem so that most real materials, including BN nanotubes, are elastically "stiff."

A second rather interesting consequence of this nonlocal theory occurs when different strain fields are separately coupled to the electric polarization. Elimination of the polarization then induces a bilinear coupling between different strain gradients. A striking consequence of the induced coupling occurs on a nanotube where both tube extension (longitudinal strain) and tube torsion (transverse strain) couple to the polarization field. Here, a uniform tensile or compressive stress induces extension or compression and a concomitant torsion in its equilibrium state. This conversion of a longitudinal to a torsion stress is impossible in a macroscopic homogeneous tube or cylinder, though it would generically oc- 
cur in the elastic theory of a helical filament. We conclude that the appearance of an anomalous elastic response of this type is a macroscopic manifestation of the underlying (atomic scale) chirality of the structure.

In this paper, we develop a theory for this anomalous elastic response and apply it to study the equilibrium strained state of a family of BN nanotubes. We find that it has a rather rich structure, depending on the relative strengths of the elastic and electrostatic interactions and on the chiral angle of the tube. Nonetheless, these anomalous couplings turn out to be fairly small and will likely be difficult to observe directly in ordinary experimental environments. Physically, this reflects the fact that finite radius BN are mechanically stiff structures that tend to be dominated by their bare elastic interactions, weakly perturbed by the electrostatic effects of the type considered here.

The rest of this paper is organized as follows. In Sec. II, we develop a continuum theory appropriate for one dimensional (1D) systems and discuss limiting cases. In Sec. III, we present exact numerical and approximate analytic solutions for the various parameter regimes. In Sec. IV, we apply our results to chiral BNNTs and calculate their piezoelectric response. Finally, in Sec. V, we summarize our results and present conclusions.

\section{FREE ENERGY FORMALISM}

We consider a piezoelectric nanotube with radius $R$ and length $L$, and let the tube axis be the $z$ axis. In what follows, we assume that $L \gg R \gg a_{0}$, where $a_{0}$ is the B-N lattice spacing. In this limit of large aspect ratio and large tube radius, a continuum theory is appropriate.

The tube is placed under a constant external stress, which we partition into its longitudinal component $f_{s}$ and torsional component $f_{t}$. The applied stresses induce strain fields $\eta_{s}(z)$ (stretch) and $\eta_{t}(z)$ (twist). The induced polarization is related to these strains through $P(z)=e_{s} \eta_{s}(z)+e_{t} \eta_{t}(z)$, where $e_{s}$ and $e_{t}$ are the linear piezoelectric constants that depend on the chiral angle $\theta$. For a general chiral nanotube, both $e_{s}$ and $e_{t}$ are nonzero. For the two high symmetry classes of nanotubes, only one piezoelectric coefficient is nonzero, $e_{s}$ for "zigzag" nanotubes and $e_{t}$ for "armchair" nanotubes. The special cases where only one elastic degree of freedom couples to the polarization have no induced cross coupling terms and were considered previously. ${ }^{4}$

The free energy of the system can be written as the sum of three terms,

$$
G=G_{\text {elastic }}+G_{\text {electro. }}-\sum_{i} \int_{0}^{L} f_{i} \eta_{i}(z) d z,
$$

where $G_{\text {elastic }}$ is the elastic contribution, $G_{\text {electro. }}$ is the electrostatic contribution, and the last term represents the work done by the applied stress, and the sum runs over $s$ and $t$. The elastic contribution is

$$
G_{\text {elastic }}=\sum_{i, j} \frac{1}{2} C_{i j} \int_{0}^{L} \eta_{i}(z) \eta_{j}(z) d z,
$$

where the indices $i$ and $j$ run over $s$ and $t$ and we have introduced four 1D elastic moduli $C_{i j}$. The diagonal 1D elas- tic moduli are radius dependent quantities with units of energy/length and are given by $C_{s s}=2 \pi R C_{11}$ and $C_{t t}=2 \pi R C_{66} . C_{11}$ and $C_{66}$ are two dimensional (2D) elastic constants in the conventional Voigt notation and are the relevant intensive variables, with units of energy/area, which depend on the nanotube chemistry. The off-diagonal term, $C_{s t}$, is small and decreases as $1 / R^{2}$ in the limit of large radius tubes. We therefore ignore this term by setting $C_{s t}=0$ in all that follows.

Using the fact that the bound charge density is related to spatial derivatives of the strains through $\rho(z)=-\sum_{i} e_{i} \partial \eta_{i}(z) / \partial z$, the electrostatic contribution to the free energy can be written as

$$
G_{\text {electro. }}=\sum_{i, j} \frac{e_{i} e_{j}}{2} \int_{0}^{L} \int_{0}^{L} \frac{\partial \eta_{i}(z)}{\partial z} V\left(z-z^{\prime}\right) \frac{\partial \eta_{j}\left(z^{\prime}\right)}{\partial z^{\prime}} d z d z^{\prime},
$$

where again the sums on $i$ and $j$ are over $s$ and $t$. Here, $V\left(z-z^{\prime}\right)$ is a Coulomb kernel describing the electrostatic interaction between rings of charge centered at positions $z$ and $z^{\prime}$ along the tube axis. $e_{s}$ and $e_{t}$ are 1D piezoelectric constants with units of charge. They may be obtained by rotating the piezoelectric constants for an infinite 2D sheet onto the symmetry axes of a tube. ${ }^{3}$ The threefold symmetry of an infinite hexagonal lattice reduces the number of independent 2D piezoelectric constants to 1 , which we take as $e_{2} \equiv e_{x x x}$ with units of charge/length. If we define the chiral angle as the angle between the nanotube axis and a $2 \mathrm{D}$ primitive translation vector $\left(\theta=30^{\circ}\right.$ is the zigzag configuration and $\theta=0^{\circ}$ is the armchair configuration), then the $1 \mathrm{D}$ piezoelectric constants are given by

$$
\begin{gathered}
e_{s}=C e_{2} \sin (3 \theta), \\
e_{t}=-C e_{2} \cos (3 \theta),
\end{gathered}
$$

where $C$ is the nanotube circumference. ${ }^{3}$ These expressions are derived neglecting finite radius corrections due to the tube curvature. However, these expressions agree with numerical results to within 15\% for the smallest nanotubes considered, with better agreement for larger radius tubes where curvature effects are reduced.

The polarization induced along the tube is composed of dipoles with both a longitudinal and an azimuthal component. The longitudinal component accounts for the electrostatic energy given in Eq. (3). The total dipole moment of the azimuthal polarization integrates to zero, but higher order moments do not vanish and may contribute to the energy. The energy from these interacting rings of dipoles is of the form

$$
G_{\text {rings }} \sim-\left(\frac{a_{0}}{R}\right)^{2} \frac{e_{i}^{\phi} e_{j}^{\phi}}{R} \iint d x d x^{\prime} \eta_{i}(x) K\left(x-x^{\prime}\right) \eta_{j}\left(x^{\prime}\right),
$$

where the integral is written in terms of the dimensionless variable $x=z / R$ and $e_{i}^{\phi}$ is the appropriate piezoelectric constant giving the local azimuthal polarization in terms of the induced strains. The important result is that the kernel goes 
asymptotically as $K(x) \sim 1 / x^{2 m+1}$, where $m$ is approximately the number of $2 \mathrm{D}$ unit cells in one tube circumference. This kernel decays so fast that we may approximate it with a delta function. This term then has the same functional form as $G_{\text {elastic }}$. However, as we will see below, the terms we will keep in $G_{\text {elastic }}$ scale with the radius as $R^{2}$, whereas all terms in $G_{\text {rings }}$ scale as $1 / R^{3}$. Since we are interested in the limit of large radius tubes, this energy represents a minor correction to the elastic energy of the system and will be ignored.

The strain field may be discontinuous at the boundaries of the nanotube. To account for this, we let $\eta_{i}(z)=g_{i}(z) H(z)$, where $g_{i}(z)$ is a continuous function of $z$ and $H(z)=1$ for $0 \leqslant z \leqslant L$ and 0 otherwise. Writing $\eta_{i}(z)$ in this form is con- venient because it allows us to keep track of the delta function contributions to $\eta_{i}^{\prime}(z)$ at the boundary of the nanotube. In particular, it removes any ambiguity that might arise in an expression such as $\eta_{i}^{\prime}(0)$. In what follows, we will call both $\eta_{i}(z)$ and $g_{i}(z)$ the strain field since they agree over the length of the tube.

We extract the dimensional dependence of the energies in Eq. (1) by expressing all lengths in units of the tube radius and all radius dependent quantities by their relevant intensive quantities. We define a scaled length $x=z / R$ and the dimensionless aspect ratio as $\lambda_{1}=L / R$. Then, after integrating the electrostatic free energy by parts, the free energy is given by

$$
\begin{aligned}
G= & \pi R^{2} \int_{0}^{\lambda_{1}} d x\left[C_{11} g_{s}^{2}(x)+C_{66} g_{t}^{2}(x)\right]-R \int_{0}^{\lambda_{1}} d x\left[f_{s} g_{s}(x)+f_{t} g_{t}(x)\right] \\
& +2 \pi^{2} \operatorname{Re}_{2}^{2} \int_{0}^{\lambda_{1}} d x d x^{\prime} \frac{\partial^{2} V\left(x-x^{\prime}\right)}{\partial x \partial x^{\prime}}\left[\sin ^{2}(3 \theta) g_{s}(x) g_{s}\left(x^{\prime}\right)-2 \cos (3 \theta) \sin (3 \theta) g_{s}(x) g_{t}\left(x^{\prime}\right)+\cos ^{2}(3 \theta) g_{t}(x) g_{t}\left(x^{\prime}\right)\right] \\
& -4 \pi^{2} \operatorname{Re}_{2}^{2} V^{\prime}(0+) \int_{0}^{\lambda_{1}} d x\left[\sin ^{2}(3 \theta) g_{s}^{2}(x)-2 \sin (3 \theta) \cos (3 \theta) g_{s}(x) g_{t}(x)+\cos ^{2}(3 \theta) g_{t}^{2}(x)\right],
\end{aligned}
$$

where $V^{\prime}(0+)$ indicates the one-sided right derivative of the Coulomb kernel. It may be that $V^{\prime}(0+)=\infty$, but there is an equal and opposite infinity in the double integral so that the energy always remains finite.

The equilibrium strain fields are obtained by minimizing $G$ with respect to the strains and satisfy

$$
\vec{g}(x)-2 V^{\prime}(0+) \Lambda \vec{g}(x)+\int_{0}^{\lambda_{1}} d x^{\prime} \frac{\partial^{2} V\left(x-x^{\prime}\right)}{\partial x \partial x^{\prime}} \Lambda \vec{g}\left(x^{\prime}\right)=\vec{\beta}
$$

For notational simplicity, we have defined a strain "vector" as $\vec{g}(x)=\left(g_{s}(x), g_{t}(x)\right)$ and an elastic limit "vector" as $\vec{\beta}=\left(\beta_{s}, \beta_{t}\right)$, where $\beta_{s}=f_{s} /\left(2 \pi R C_{11}\right)$ is the value of the uniaxial strain in the absence of piezoelectric effects and $\beta_{t}=f_{t} /\left(2 \pi R C_{66}\right)$ is the value of the torsional strain in the absence of piezoelectric effects. The matrix $\Lambda$ is given by

$$
\Lambda=2 \pi\left(\begin{array}{cc}
\lambda_{2} \sin ^{2}(3 \theta) & -\lambda_{2} \sin (3 \theta) \cos (3 \theta) \\
-\lambda_{3} \sin (3 \theta) \cos (3 \theta) & \lambda_{3} \cos ^{2}(3 \theta)
\end{array}\right),
$$

where we have introduced two dimensionless constants, $\lambda_{2}=e_{2}^{2} /\left(R C_{11}\right)$ and $\lambda_{3}=e_{2}^{2} /\left(R C_{66}\right)$, which measure the relative strengths of the electrostatic and elastic response. Note that $C_{11}$ and $C_{66}$ are generally of the same order of magnitude, so $\lambda_{2}$ and $\lambda_{3}$ will also be of the same order of magnitude.

We will see below that the solutions to Eq. (7) asymptotically approach the elastic limit with a characteristic decay length controlled by $\lambda_{2}$ and $\lambda_{3}$. There are two limiting cases of interest. When the tube is much longer than the decay length $\left(\lambda_{1} \gg 2 \pi \lambda_{2}, 2 \pi \lambda_{3}\right)$, the strains are near their elastic limits over most of the tube length, with deviations from the elastic limit near the tube ends. Such systems are dominated by their elastic response to the applied stress, and we call this the elastically dominated limit. Conversely, when the tube is much shorter than the decay length $\left(\lambda_{1} \ll 2 \pi \lambda_{2}, 2 \pi \lambda_{3}\right)$, the strains never decay to their elastic limits. Such systems are dominated by their electrostatic response to the applied stress, and we call this the electrostatically dominated limit. Solutions for these two limiting cases will be explored in the next section.

The matrix $\Lambda$ is not invertible; therefore, the strains are not linearly independent. We find that the strains are related by

$$
g_{t}(x)=\beta_{t}+\frac{\lambda_{3} \cos (3 \theta)}{\lambda_{2} \sin (3 \theta)}\left[\beta_{s}-g_{s}(x)\right]
$$

Plugging this relationship into Eq. (7) allows us to write the equilibrium equation in terms of $g_{s}(x)$ alone,

$$
\begin{aligned}
g_{s}(x)= & \beta_{s}-2 V^{\prime}(0+)\left[\gamma g_{s}(x)-\tau\right] \\
& +\int_{0}^{\lambda_{1}} d x^{\prime} \frac{\partial^{2} V\left(x-x^{\prime}\right)}{\partial x \partial x^{\prime}}\left[\gamma g_{s}\left(x^{\prime}\right)-\tau\right],
\end{aligned}
$$

where we have defined two new constants, 


$$
\gamma=2 \pi\left[\lambda_{2} \sin ^{2}(3 \theta)+\lambda_{3} \cos ^{2}(3 \theta)\right]
$$

and

$$
\tau=2 \pi \cos (3 \theta)\left[\lambda_{3} \cos (3 \theta) \beta_{s}+\lambda_{2} \sin (3 \theta) \beta_{t}\right] .
$$

In order to solve Eq. (10), an appropriate Coulomb kernel must be specified. In the simplest continuum model where the kernel describes the interaction of charged rings at positions $x$ and $x^{\prime}$ on a tube, the Coulomb kernel is given by

$$
V\left(x-x^{\prime}\right)=\frac{1}{2 \pi} \int_{0}^{2 \pi} \frac{d \theta}{\sqrt{\left(x-x^{\prime}\right)^{2}+\sin ^{2}(\theta)+[1-\cos (\theta)]^{2}}} .
$$

This kernel diverges logarithmically as $x \rightarrow x^{\prime}$ and leads to an infinite energy for surface charges. Using this kernel, the boundary condition on the strain fields is $e_{s} \eta_{s}(0)+e_{t} \eta_{t}(0)$ $=0$. A divergent energy is an artifact of our continuum model and signals the breakdown of the model at short distances. It is useful to replace the divergent kernel with a softened kernel,

$$
V_{\text {soft }}\left(x-x^{\prime}\right)=\frac{1}{\left|x-x^{\prime}\right|+\alpha},
$$

which retains the correct long-range behavior but remains finite as $x \rightarrow x^{\prime}$. This kernel allows the presence of a nonzero surface charge, with the "cost" of a surface charge controlled by the cutoff $\alpha$. The tube radius forms a natural length scale below which the continuum model breaks down, suggesting that $\alpha \approx 1$ is an appropriate cutoff. In Ref. 4, it was shown that using the softened kernel with $\alpha=1$ gave nearly identical results for the strain, charge density, and potential across the tube as those obtained using the divergent kernel. The only notable difference is that the softened kernel gives a nonzero surface charge which is replaced by a logarithmically divergent bound charge density at the boundaries with no surface charge for the divergent kernel. These are indistinguishable for all practical purposes.

Once the charge density is computed, the potential along the tube is determined by

$$
U(z)=\int_{0}^{L} V\left(z-z^{\prime}\right) \rho\left(z^{\prime}\right) d z^{\prime} .
$$

After expressing all lengths in terms of the tube radius and integrating by parts, the potential becomes

$$
U(x)=\sum_{i} \frac{e_{i}}{R} \int_{0}^{\lambda_{1}} g_{i}\left(x^{\prime}\right) \frac{\partial V\left(x-x^{\prime}\right)}{\partial x^{\prime}} d x^{\prime} .
$$

Differentiating this expression and writing all constants in terms of intensive variables allow us to relate the potential to the strain through

$$
\frac{\partial U(x)}{\partial x}=\frac{e_{2}}{\lambda_{2} \sin (3 \theta)}\left[\beta_{s}-g_{s}(x)\right] .
$$

Integrating this expression gives the potential as an integral over the strain field,

$$
U(x)=\frac{e_{2}}{\lambda_{2} \sin (3 \theta)} \int_{x}^{\lambda_{1} / 2} d x^{\prime}\left(g_{s}\left(x^{\prime}\right)-\beta_{s}\right)
$$

where we have set the potential to zero at the middle of the tube.

Once $g_{t}(z)$ is obtained through Eq. (9), the change in the azimuthal angle $\phi$ at a point $z$ is given by $R d \phi=g_{t}(z) d z$. The total twist induced along the tube is therefore given by

$$
\Delta \phi=\int_{0}^{\lambda_{1}} d x g_{t}(x),
$$

where we have transformed to the scaled length. Following the usual convention, the total twist is positive if the tube rotates in the counterclockwise direction and negative if it rotates in the clockwise direction. Notice from Eq. (9) that for a general chiral tube, there is a coupling between longitudinal and torsional strains such that a uniaxial stress alone $\left(\beta_{t}=0\right)$ is sufficient to induce twisting in the nanotube. This coupling vanishes in the high symmetry zigzag and armchair nanotubes, as may be seen by writing Eq. (9) as $\lambda_{2} \sin (3 \theta)\left[g_{t}(x)-\beta_{t}\right]=\lambda_{3} \cos (3 \theta)\left[\beta_{s}-g_{s}(x)\right]$. For armchair nanotubes, $\sin (3 \theta)=0$, which forces $g_{s}(x)=\beta_{s}$ but sets no restrictions on $g_{t}(x)$. Likewise, for zigzag nanotubes, $\cos (3 \theta)=0$, which forces $g_{t}(x)=\beta_{t}$ but sets no restrictions on $g_{s}(x)$. For a general chiral tube, the origin of this "stretchtwist" coupling lies in the electrostatic term in the free energy. In regions where one flavor of strain has large spatial gradients, the bound charge density due to that flavor of strain is also large. The overall bound charge density may be lowered in that region by gradients in the other flavor of strain that produce bound charges of opposite sign. The equilibrium strain fields are those that create a bound charge density profile that gives the lowest electrostatic free energy consistent with Eqs. (9) and (10).

If we consider a system with $\beta_{t}=0$, then Eqs. (9) and (19) give

$$
\Delta \phi=C \frac{\cos (3 \theta)}{\sin (3 \theta)},
$$

where $C=\left(\lambda_{3} / \lambda_{2}\right) \int\left[\beta_{s}-g_{s}(x)\right] d x$. We will see below that for a system under tensile stress, $\beta_{s} \geqslant g_{s}(x)$ for all $x$. In this case, $C \geqslant 0$, and the sign of the total induced twist in the nanotube is entirely determined by the sign of the chiral angle $(\bmod 2 \pi / 3)$. We find that $\Delta \phi>0$ for right-handed nanotubes and $\Delta \phi<0$ for left-handed nanotubes. Therefore, the sign of the induced twist may be used to determine the handedness of a chiral piezoelectric nanotube. There is no known order parameter for the handedness of a chiral nanotube that can be built up out of the two primitive vectors of the $2 \mathrm{D}$ hexagonal lattice and the translational vector of the nanotube. As such, an unambiguous assignment of nanotube handedness is difficult with structural information alone. A measurement of the sign of the stretch-twist coupling allows one to easily make this assignment.

A nonpiezoelectric chiral nanotube will also couple stretch and twist to some degree due to the rearrangement of the atomic basis upon stretching. This effect is absent in our 


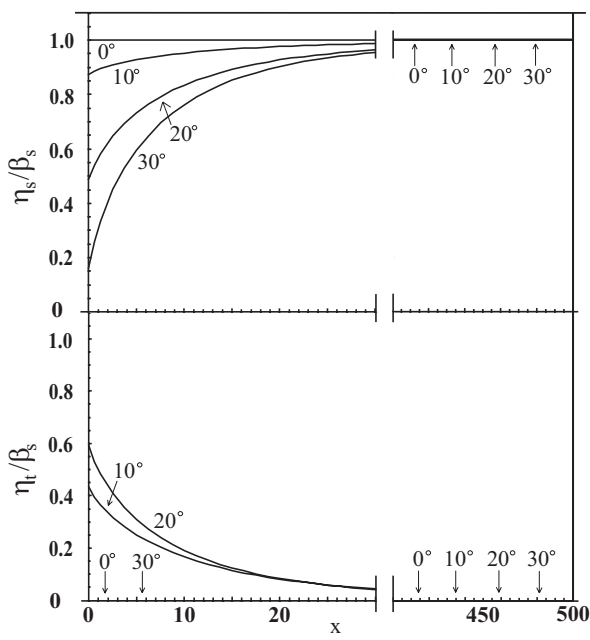

FIG. 1. Equilibrium solutions for a piezoelectric tube in the elastically dominated limit using the soft kernel. The upper panel shows the induced uniaxial strain for tubes of various chiralities, while the lower panel shows the corresponding induced torsional strains. The graph is broken along the $x$ axis in order to show details near the tube boundary. The graphs were generated using $\beta_{s}=1$, $\beta_{t}=0, \lambda_{1}=1000, \lambda_{2}=10 / \pi, \lambda_{3}=2 \lambda_{2}$, and $e_{2}=\sqrt{5} / \pi$ (charge/length).

formalism because we have set $C_{s t}=0$, when in reality this quantity is small but nonzero for a chiral nanotube. It is worth noting that the electrostatically induced stretch-twist coupling described above is in general several orders of magnitude larger than the coupling in nonpiezoelectric nanotubes. The torsional strain induced in a piezoelectric system can easily be of the same order of magnitude as the uniaxial strain.

\section{EQUILIBRIUM SOLUTIONS}

The equilibrium solutions for the strain fields $g_{i}(z)$ [Eqs. (9) and (10)], the bound charge density $\rho(z)=-\Sigma_{i} e_{i} \partial \eta_{i}(z) / \partial z$, and the electrostatic potential $U(z)$ [Eq. (18)] were determined using finite element analysis to solve Eq. (10). Convergence of the solution was monitored by increasing the number of finite elements in increments of 100 until the difference in successive solutions was less than $1 \%$ at the end of the tube where the convergence is the slowest.

\section{A. Elastically dominated limit}

The elastically dominated limit is defined by the condition that $2 \pi \lambda_{2}, 2 \pi \lambda_{3} \ll \lambda_{1}$. We investigate the case of a tube under constant uniaxial stress in the absence of torsional stress. The equilibrium strain fields for several chiral angles are presented in Fig. 1. The strain fields rapidly relax to their elastic limits and over much of the tube $\eta_{s}(x) \approx \beta_{s}$ and $\eta_{t}(x) \approx 0$. Near the boundaries of the tube, the electrostatic interactions effectively increase $C_{11}$, suppressing the longitudinal strain. Importantly, notice that for chiral tubes, $\eta_{t}(x) \neq 0$ for all $x$ even though $\beta_{t}=0$. Because the longitudinal and torsional strains couple through spatial derivatives,

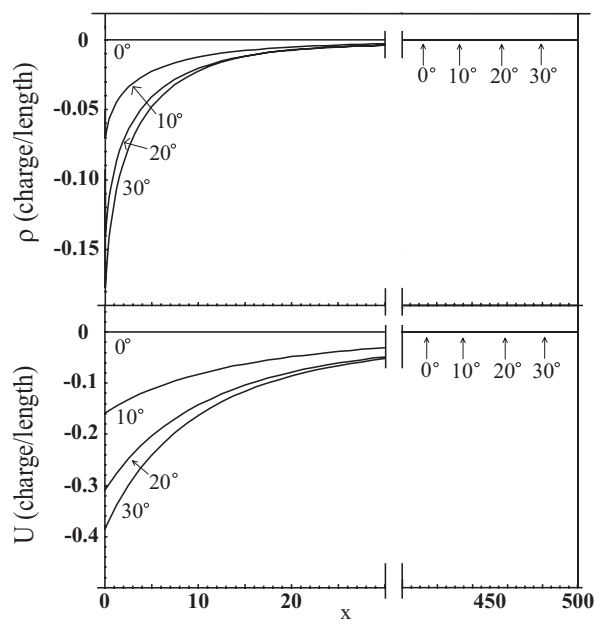

FIG. 2. Equilibrium solutions for a piezoelectric tube in the elastically dominated limit using the soft kernel. The upper panel shows the induced bound charge density for various chiral angles and the lower panel shows the corresponding electrostatic potential along the tube. The graph is broken along the $x$ axis in order to show details near the tube boundary. The graphs were generated using $\beta_{s}=1, \beta_{t}=0, \lambda_{1}=1000, \lambda_{2}=10 / \pi, \lambda_{3}=2 \lambda_{2}$, and $e_{2}=\sqrt{5} / \pi$ (charge/length).

the chiral tubes develop some nonzero twist near the tube ends. The total twist induced in the tube reaches a maximum at $\theta=15^{\circ}$ and goes to zero as $\theta \rightarrow 0^{\circ}$ or $\theta \rightarrow 30^{\circ}$.

The bound charge density and the electrostatic potential are plotted in Fig. 2. The charge density is localized at the ends of the tube and decays rapidly on the interior. Not shown are delta functions in the charge density right at the tube boundary. These are surface charges which appear because the polarization is nonzero at the tube boundary with the soft kernel. The potential varies rapidly near the tube ends due to the presence of the induced bound charges. On the interior of the tube, the potential varies slowly and resembles the electrostatic potential produced by two opposite point charges at the ends of the tube. Almost the entire potential drop across the tube occurs near the tube boundaries.

Both the bound charge density and the potential are maximized by letting $\theta=30^{\circ}$ and decrease monotonically as $\theta \rightarrow 0$. We find that the total potential difference across the tube is well fitted by $\Delta U \sim \sin (3 \theta)$. Therefore, under longitudinal stress, the piezoelectric response is insensitive to small perturbations around the zigzag configuration.

A striking feature of Figs. 1 and 2 is that the effects of the chirality only manifest themselves at the ends of the tube. The bulk of the tube responds to an applied stress as an ordinary elastic medium. This is a somewhat surprising result because it implies that boundaries are necessary for chiral effects to appear and that the bulk of the tube has no "knowledge" of its own chirality. Although this is evident from our free energy formalism, an explanation of this effect based on the symmetry properties of the tube is desirable and is the subject of current investigations.

An approximate analytic solution to Eq. (10) may be obtained with a perturbation expansion and gives 


$$
g_{s}(x)=\beta_{s}-\left(\gamma \beta_{s}-\tau\right)\left[\frac{1}{(x+\alpha)^{2}}+\frac{1}{\left(\lambda_{1}-x+\alpha\right)^{2}}\right] .
$$

This expression always gives the correct asymptotic decay to the elastic limit far from the tube ends. If the system is very far into the elastic limit, so that $\left(2 \pi \lambda_{2} / \alpha^{2}\right) \ll 1$, then this expression holds over the entire length of the tube. Using Eq. (18) to compute the potential difference, $\Delta U=U\left(\lambda_{1}\right)-U(0)$, across a tube under uniaxial stress only gives

$$
\Delta U=C_{U}-\frac{4 \pi e_{2} \sin (3 \theta) \beta_{s}}{\lambda_{1}+\alpha} .
$$

The constant $C_{U}$ can be determined numerically. If the system is extremely elastic $\left[\left(2 \pi \lambda_{2} / \alpha^{2}\right) \ll 1\right]$, then $C_{U}=4 \pi e_{2} \sin (3 \theta) \beta_{s} / \alpha$.

We may use Eq. (9) to obtain $g_{t}(x)$ from $g_{s}(x)$, and then compute the total twist along the tube using Eq. (19). For a tube under uniaxial stress only, this gives

$$
\Delta \phi=C_{\phi}-\frac{4 \pi \lambda_{3} \sin (3 \theta) \cos (3 \theta) \beta_{s}}{\lambda_{1}+\alpha} .
$$

The constant $C_{\phi}$ can be determined numerically. If the system is extremely elastic $\left[\left(2 \pi \lambda_{2} / \alpha^{2}\right) \ll 1\right]$, then $C_{\phi}=4 \pi \lambda_{3} \sin (3 \theta) \cos (3 \theta) \beta_{s} / \alpha$.

Equations (22) and (23) show that, for systems in the elastic limit, the induced potential and the total twist essentially do not scale with tube length. This is a consequence of the fact that the bound charge density is localized near the tube ends with a decay length set by material parameters, independent of length.

\section{B. Electrostatically dominated limit}

The electrostatically dominated limit is defined by the condition $2 \pi \lambda_{2}, 2 \pi \lambda_{3} \gg \lambda_{1}$. Again, we investigate a tube under uniaxial stress only $\left(\beta_{t}=0\right)$. In Fig. 3 , we plot the equilibrium strain fields for several chiral angles. Note that in this case, the tube is shorter than the strain decay length, and the strain fails to relax to its elastic limit. The tube develops significant torsional strain along its entire length unless it is in one of the high symmetry configurations.

The bound charge density and the electrostatic potential in the electrostatic limit are plotted in Fig. 4 for several chiral angles. The bound charge density is nearly linear throughout the interior of the tube and vanishes at the middle of the tube by symmetry. Near the boundaries, the charge density begins to develop a logarithmic divergence, which is cut off because of the soft kernel. Not shown are delta functions in the charge density right at the tube boundary. Again, these are surface charge densities that arise due to our use of the soft kernel. The potential is also nearly linear along the entire length of the tube and vanishes at the center due to symmetry. Near the boundaries, there are small, nondivergent corrections to the linear behavior. A linear electrostatic potential profile is what we would naively expect from the conventional three dimensional (3D) case, but note the remarkably

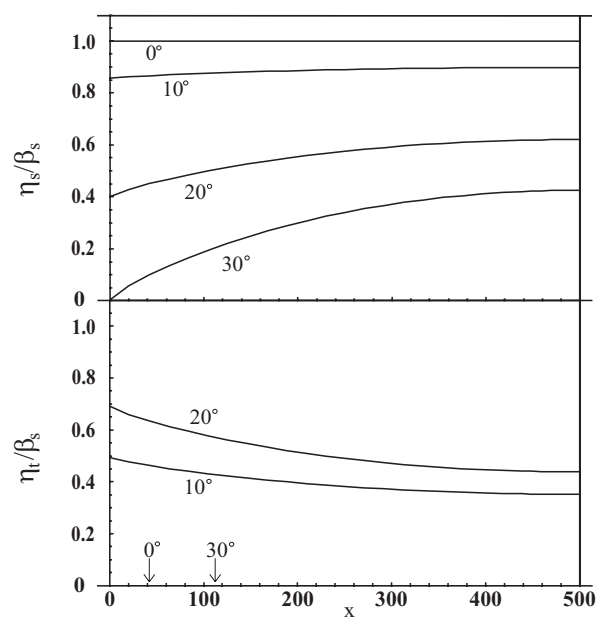

FIG. 3. Equilibrium solutions for a piezoelectric tube in the electrostatically dominated limit using the soft kernel. The upper panel shows the induced uniaxial strain for tubes of various chiralities, while the lower panel shows the corresponding induced torsional strains. The graphs were generated using $\beta=1, \lambda_{1}=1000$, $\lambda_{2}=10000 / \pi, \lambda_{3}=2 \lambda_{2}$, and $e_{2}=\sqrt{5000} / \pi$ (charge/length).

different conditions which give rise to the linear potential in this case. In the 3D case, the linear potential is produced by true surface charges interacting via a long-range electrostatic interaction. In this 1D case, the linear potential is produced by a nearly linear bound charge density interacting via a short-range electrostatic interaction.

Inspection of the strains in Fig. 3 shows that they may be well fitted by quadratic curves. An approximate analytic solution may be obtained in the form $g_{s}(x) \approx c_{o}+c_{2}\left(x-\lambda_{1} / 2\right)^{2}$, where

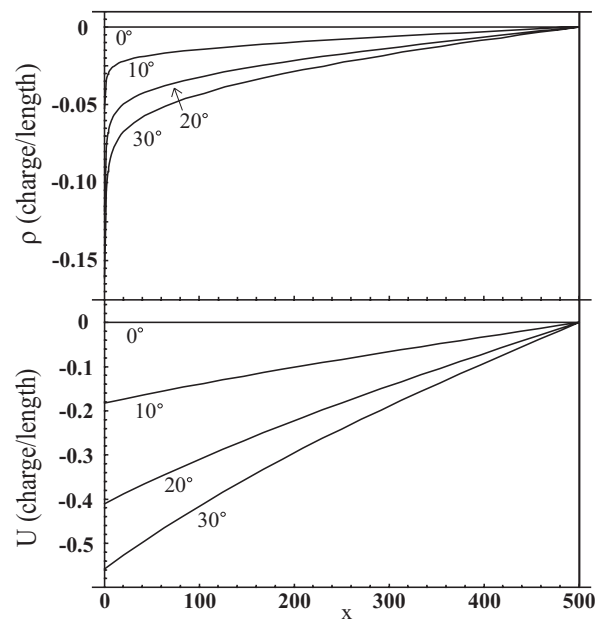

FIG. 4. Equilibrium solutions for a piezoelectric tube in the electrostatically dominated limit using the soft kernel. The upper panel shows the induced bound charge density for tubes of various chiralities, while the lower panel shows the corresponding electrostatic potential along the tube. The graphs were generated using $\beta=1, \quad \lambda_{1}=1000, \quad \lambda_{2}=10000 / \pi, \quad \lambda_{3}=2 \lambda_{2}, \quad$ and $\quad e_{2}=\sqrt{5000} / \pi$ (charge/length). 


$$
\begin{gathered}
c_{0}=\left(\frac{1}{D}\right)\left\{\frac{\beta_{s} \lambda_{1}^{6}}{180}+\left(5 \beta_{s} \gamma-\tau\right)\left[\frac{\lambda_{1}^{5}}{60 \alpha}+O\left(\lambda_{1}^{4} \log \lambda_{1}\right)\right]\right. \\
\left.+\gamma \tau\left[\frac{4 \lambda_{1}^{3}}{3 \alpha} \log \left(\frac{\lambda_{1}}{\alpha}\right)-\frac{28 \lambda_{1}^{3}}{9}+O\left(\lambda_{1}^{2} \log \lambda_{1}\right)\right]\right\}, \\
c_{2}=\left(\frac{\tau-\beta_{s} \gamma}{D}\right)\left[\frac{\lambda_{1}^{3}}{3 \alpha}+O\left(\lambda_{1}^{2} \log \lambda_{1}\right)\right],
\end{gathered}
$$

and

$$
\begin{aligned}
D= & \frac{\lambda_{1}^{6}}{180}+\gamma\left[\frac{\lambda_{1}^{5}}{15 \alpha}+O\left(\lambda_{1}^{4}\right)\right]+\gamma^{2}\left[\frac{4 \lambda_{1}^{3}}{3 \alpha} \log \left(\frac{\lambda_{1}}{\alpha}\right)-\frac{28 \lambda_{1}^{3}}{9}\right. \\
& \left.+O\left(\lambda_{1}^{2} \log \lambda_{1}\right)\right]
\end{aligned}
$$

This expansion is accurate to within a few percent provided that $\gamma, \tau \gg \lambda_{1}$, which is generally true if $\lambda_{2}, \lambda_{3} \gg 1$. The exception occurs if $\theta \rightarrow \pi / 6$, in which case $\tau \rightarrow 0$ for any $\lambda_{2}$, $\lambda_{3}$. Even in this case, the expansion differs from the numerical results only near the tube ends and still gives acceptable agreement with the numerical results for the potential and total twist. For the potential across the tube, we find

$$
\Delta U=\left(\beta_{s}-c_{0}\right) \lambda_{1}-\frac{c_{2}}{12} \lambda_{1}^{3} .
$$

Because $\left(\beta_{s}-c_{0}\right) \gg c_{2}$, the potential scales nearly linearly with tube length, an effect anticipated from our numerical results. The total twist along the nanotube will also have a nearly linear scaling with length, provided that the system remains in the electrostatic regime.

\section{BORON NITRIDE NANOTUBES}

We now apply our theory to calculate the piezoelectric response of a BNNT under uniform longitudinal tensile stress. The $2 \mathrm{D}$ elastic constants of a $\mathrm{BN}$ sheet are given by $\mathrm{y}^{5,6}$ $C_{11} \approx 0.30 \mathrm{TPanm}$ and $C_{66} \approx 0.10 \mathrm{TPa} \mathrm{nm}$, and the piezoelectric constant is given by ${ }^{3} e_{2}=0.12 \mathrm{e} / \mathrm{bohr}$. Experimentally, accessible forces are on the nanonewton scale; we arbitrarily assume a force of $1 \mathrm{nN}$. Typical tube dimensions are $R=1 \mathrm{~nm}$ and $L=1 \mu \mathrm{m}$.

Using these values, we find that $\lambda_{1}=1000, \lambda_{2}$ $=4.4 \times 10^{-3}, \lambda_{3}=1.3 \times 10^{-2}, \beta_{s}=5.3 \times 10^{-4}$, and $\beta_{t}=0$. These numbers show that BNNTs are an extremely elastically dominated system, and our perturbation formulas given in Sec. III A apply. Equation (21) agrees with the numerical results for the strain to better than $0.1 \%$, and
Eqs. (22) and (23), with $C_{U}=4 \pi e_{2} \sin (3 \theta) \beta_{s} / \alpha$ and $C_{\phi}=4 \pi \lambda_{3} \sin (3 \theta) \cos (3 \theta) \beta_{s} / \alpha$, give the electrostatic potential and total twist with even better agreement.

For all chiral angles, the strain fields decay to within $0.5 \%$ of their elastic limits at a depth of $2 R$ and are always within $3 \%$ of the elastic limit. The interior of the tube develops no significant twist and essentially responds as an achiral nanotube. The total twist, in radians, induced in the system as a function of chiral angle is $\Delta \phi(\theta)=8.7 \times 10^{-5} \sin (3 \theta) \cos (3 \theta)$. The system develops significant surface charges and a bound charge density that decays rapidly on the interior of the tube, and the entire potential drop occurs within a few radii of the tube ends. Using the values from above and converting to SI units gives the potential across the tube as a function of the chiral angle as $U(\theta)=23.0 \sin (3 \theta) \mathrm{mV}$.

Based on these calculations, BNNTs should develop a rather weak piezoelectric response with almost no induced twist regardless of chirality. Physically, this is explained by the exceptionally large elastic constants of the BN sheet, which are inherited by the nanotube and make it a stiff system. BNNTs are so elastically dominated that the predicted strain decay lengths, between 1 and 2 tube radii, place this system near the limits of what can be described by our continuum theory.

\section{CONCLUSION}

In this paper, we have developed and solved a continuum theory to describe the piezoelectric effect in 1D tubular systems. These systems exhibit different physical properties than their bulk counterparts. Unconventional physical effects include the development a bound charge density near the tube ends and a nonlinear electrostatic potential profile along the tube. Furthermore, the piezoelectric response of these systems couples longitudinal strain and torsional strains. This coupling represents a degree of freedom which may be important in nanotube characterization and nanoscale device applications.

It would be interesting to measure the nonlinear potential variation along a nanotube to verify the unconventional piezoelectric effects described here. BNNTs are the model physical system in which to realize these effects. However, BNNTs tend to be stiff structures for which the electrostatic contributions to the elastic properties are expected to be small.

\section{ACKNOWLEDGMENT}

This work was supported by the Department of Energy under Grant No. DE-FG-84ER45118.
${ }^{1}$ E. J. Mele and P. Král, Phys. Rev. Lett. 88, 056803 (2002).

${ }^{2}$ S. M. Nakhmanson, A. Calzolari, V. Meunier, J. Bernholc, and M.

B. Nardelli, Phys. Rev. B 67, 235406 (2003).

${ }^{3}$ N. Sai and E. J. Mele, Phys. Rev. B 68, 241405(R) (2003).

${ }^{4}$ P. J. Michalski, N. Sai, and E. J. Mele, Phys. Rev. Lett. 95,
116803 (2005).

${ }^{5}$ E. Hernández, C. Goze, P. Bernier, and A. Rubio, Phys. Rev. Lett. 80, 4502 (1998).

${ }^{6}$ L. Duclaux, B. Nysten, J.-P. Issi, and A. W. Moore, Phys. Rev. B 46, 3362 (1992). 